PSS $\quad \begin{aligned} & \text { PROCEEDINGS } \\ & \text { OF SCIENCE }\end{aligned}$

\title{
ATLAS Forward Detectors
}

\section{Andrew Brandt* ${ }^{* \dagger}$}

University of Texas, Arlington

E-mail: brandta@uta.edu

A brief description of the ATLAS forward detectors is given.

XVIII International Workshop on Deep-Inelastic Scattering and Related Subjects April 19-23, 2010

Convitto della Calza, Firenze, Italy

\footnotetext{
* Speaker.

${ }^{\dagger}$ For the ATLAS Collaboration (http://atlas.web.cern.ch/Atlas/Collaboration/)
} 


\section{Introduction}

The ATLAS detector [1] is optimized for large momentum transfer central physics, but also includes several forward detector systems. These include the Minimum Bias Trigger Scintillators (MBTS), the Luminosity Cherenkov Integrating Detector, the Zero-Degree Calorimeters (ZDC), and the ALFA (Absolute Luminosity for ALFA) Roman pots. Figure 1 shows the location of each of these detector systems relative to the ATLAS central detector (only one arm is shown, but all the detector systems are symmetrically located on each side of the interaction region). In the following sections these existing forward detector sub-systems, and the proposed ATLAS Forward Proton (AFP) detector will be briefly summarized. The physics goals of the detectors is covered in Christophe Royon's contribution to these proceedings.

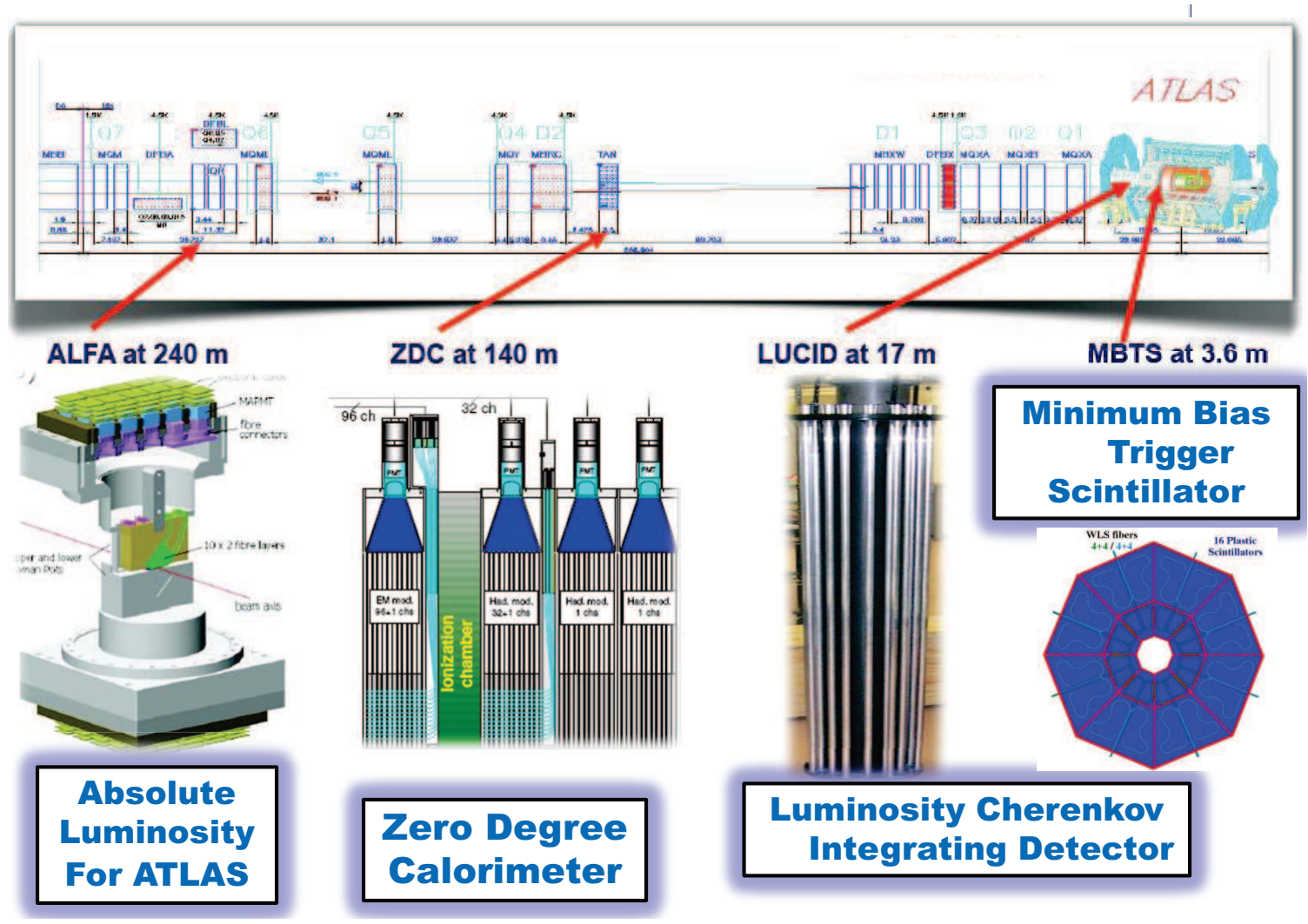

Figure 1: The layout of the approved ATLAS Forward detectors as described in the text.

\section{Approved Forward Detectors}

\subsection{MBTS}

The Minimum Bias Trigger Scintillators (MBTS) were designed to provide a Level 1 minimum bias trigger for the early data taking period. The MBTS consists of two sets of sixteen scintillation counters which are installed on the inner face of the end-cap calorimeter cryostats at $\pm 3.6 \mathrm{~m}$ from the interaction point. Each set of counters is segmented in eight units in azimuth $(\phi)$ and two 
units in pseudorapidity $(\eta)$. The inner ring of counters covers radii between $153 \mathrm{~mm}$ and $426 \mathrm{~mm}$, corresponding to the region $2.82<|\eta|<3.84$ and the outer ring covers radii between $426 \mathrm{~mm}$ and $890 \mathrm{~mm}$, corresponding to the region $2.09<|\eta|<2.82$. The scintillators are read out by TileCal PMTs connected to the standard TileCal " 3 in 1" cards. The 3 in 1 cards provides 3 outputs: a trigger signal, a readout signal, and an amplified readout signal (x64 gain). The trigger output for all TileCal modules except the MBTS operate at unit gain. During TileCal refurbishment the MBTS 3in1 channels were modified such that the MBTS trigger signal is produced with x64 output. The MBTS has been functioning as designed and has been extremely valuable in early data taking, including a critical role in the first ATLAS physics publication using $900 \mathrm{GeV}$ data [2].

\subsection{LUCID}

LUCID is a Cherenkov light detector primarily dedicated to online luminosity monitoring. LUCID is a relative luminosity detector. Its main purpose is to detect inelastic proton-proton scattering in the forward direction, in order to both measure the integrated luminosity and to provide online monitoring of the instantaneous luminosity and beam conditions.

LUCID consists of twenty aluminium tubes which surround the beam pipe and point toward the interaction point. The $1.5 \mathrm{~m}$ long mechanically polished tubes with a diameter of $15 \mathrm{~mm}$ are placed in a light-weight aluminium gas vessel which ensures that the tubes are filled with $\mathrm{C}_{4} \mathrm{~F}_{10}$ gas at a constant pressure of about 1.2 bar, providing a Cerenkov threshold of $2.8 \mathrm{GeV}$ for pions and $10 \mathrm{MeV}$ for electrons. The two LUCID detectors are installed in the ATLAS end-cap regions, at a distance of approximately $\pm 17 \mathrm{~m}$ from the interaction point, at a radial distance of approximately $10 \mathrm{~cm}$ from the beam line, and cover $5.61<|\eta|<5.93$. The Cherenkov light is read out by the radiation hard Hamamatsu R762 photomultiplier tube. The LUCID detector was optimized for high luminosity, but performed very well in early data taking with a luminosity of less than $10^{27}$ $\mathrm{cm}^{-2} \mathrm{~s}^{-1}$.

\subsection{ZDC}

The primary purpose of the ATLAS Zero-Degree Calorimeters (ZDC) is to detect forward neutrons and photons with $|\eta|>8.3$ in both proton-proton and heavy-ion collisions. The ZDCs play a key role in determining the centrality of heavy ion collisions and contribute to minimum bias and diffractive physics in proton-proton collisions. With a time resolution of about $100 \mathrm{ps}$, a coincidence of the two ZDCs can be used to greatly reduce backgrounds from beam-gas and beamhalo effects. The ZDC's reside in a slot in the TAN (Target Absorber Neutral) absorber, which would otherwise contain inert copper bars as shielding. The TAN is located at $\pm 140 \mathrm{~m}$ from the interaction point, at the location where the straight section of the beam pipe is divided into two independent beam pipes.

When the ZDCs are fully installed there will be four modules on each arm: one electromagnetic (EM) module (about 29 radiation lengths thick), and 3 hadronic modules (each about 1.14 interaction lengths thick). The modules are composed of tungsten with an embedded matrix of quartz rods which are read out by photomultiplier tubes. The modules are $9 \mathrm{~cm}$ by $9 \mathrm{~cm}$ laterally to the beam, and $15 \mathrm{~cm}$ thick. The hadronic modules are installed, while the EM modules will be installed after LHCf is removed. The hadronic modules have been integrated into the DAQ and have been functioning as designed. 


\subsection{ALFA}

The ALFA Roman pots and detectors are located at $240 \mathrm{~m}$ from the interaction point with a purpose of measuring elastic scattering at small angles in the Coulomb-Nuclear Interference region. The system consists of four Roman pot stations, two on each side of the interaction point, each housing two vertically movable detectors. The Roman pots house scintillating fiber detectors arranged in $20 \mathrm{u} / \mathrm{v}$ detection planes, providing a spatial resolution of about $30 \mu \mathrm{m}$. The luminosity calibration will be obtained during special runs using a dedicated high $\beta^{*}$ optics which features a parallel-to-point focusing to allow for a direct reconstruction of the scattered proton kinematics. The infrastructure and part of the mechanics have been installed in the LHC tunnel, while the detectors will undergo a final test beam in summer 2010, and be installed along with the final mechanics at the first available opportunity.

\section{Proposed Forward detectors}

\subsection{AFP}

The ATLAS Forward Proton (AFP) project is a proposal to upgrade the forward region at ATLAS by installing silicon and fast timing detectors $220 \mathrm{~m}$ and $420 \mathrm{~m}$ from the interaction point to detect intact final state protons scattered at small angles and with small momentum loss. The capability to detect both outgoing protons in diffractive and photoproduction processes allows for a rich QCD, electroweak, Higgs and beyond the Standard Model experimental program that has the potential to make unique measurements at the LHC. The installation of forward proton detectors at $420 \mathrm{~m}$ around both ATLAS and CMS was investigated in detail by the FP420 R\&D Collaboration in [3]. At $220 \mathrm{~m}$, a similar system to that developed for FP420 will be implemented. The $220 \mathrm{~m}$ region is less demanding from an engineering perspective since a cryogenic bypass is not required.

At both the $420 \mathrm{~m}$ and $220 \mathrm{~m}$ locations, movable beam pipes that house silicon tracking and fast timing detectors and can be remotely positioned close (a few $\mathrm{mm}$ ) to the circulating beams would be installed (see Fig. 2). The system functions as four independent momentum spectrometers, using the LHC dipole and quadrupole magnets to bend the scattered protons out of the beam envelope. It is necessary to measure both the distance from the beam and the angle of the proton tracks relative to the beam with high precision in order to reconstruct the mass of the central system produced in ATLAS. The $420 \mathrm{~m}$ stations can detect protons with fractional momentum losses in the range $0.002<\xi<0.02$. The $220 \mathrm{~m}$ stations provide complementary coverage in the range $0.02<\xi<0.2$. For events in which both protons are tagged, this corresponds to a greater than $40 \%$ acceptance over a range in central masses from $\sim 80 \mathrm{GeV}$ up to beyond $1 \mathrm{TeV}$. At luminosities in excess of $2 \times 10^{33} \mathrm{~cm}^{-2} \mathrm{~s}^{-1}$ it is necessary to measure the time of arrival of the protons with an accuracy of 20 to 30 ps to suppress overlap backgrounds. As the luminosity approaches $10^{34} \mathrm{~cm}^{-2} \mathrm{~s}^{-1}$, a resolution of $10 \mathrm{ps}$ or better become necessary to for certain physics topics. The AFP letter of intent has been reviewed by ATLAS, and the proponents have been encouraged to pursue a Technical Proposal addressing the outstanding technical issues. 


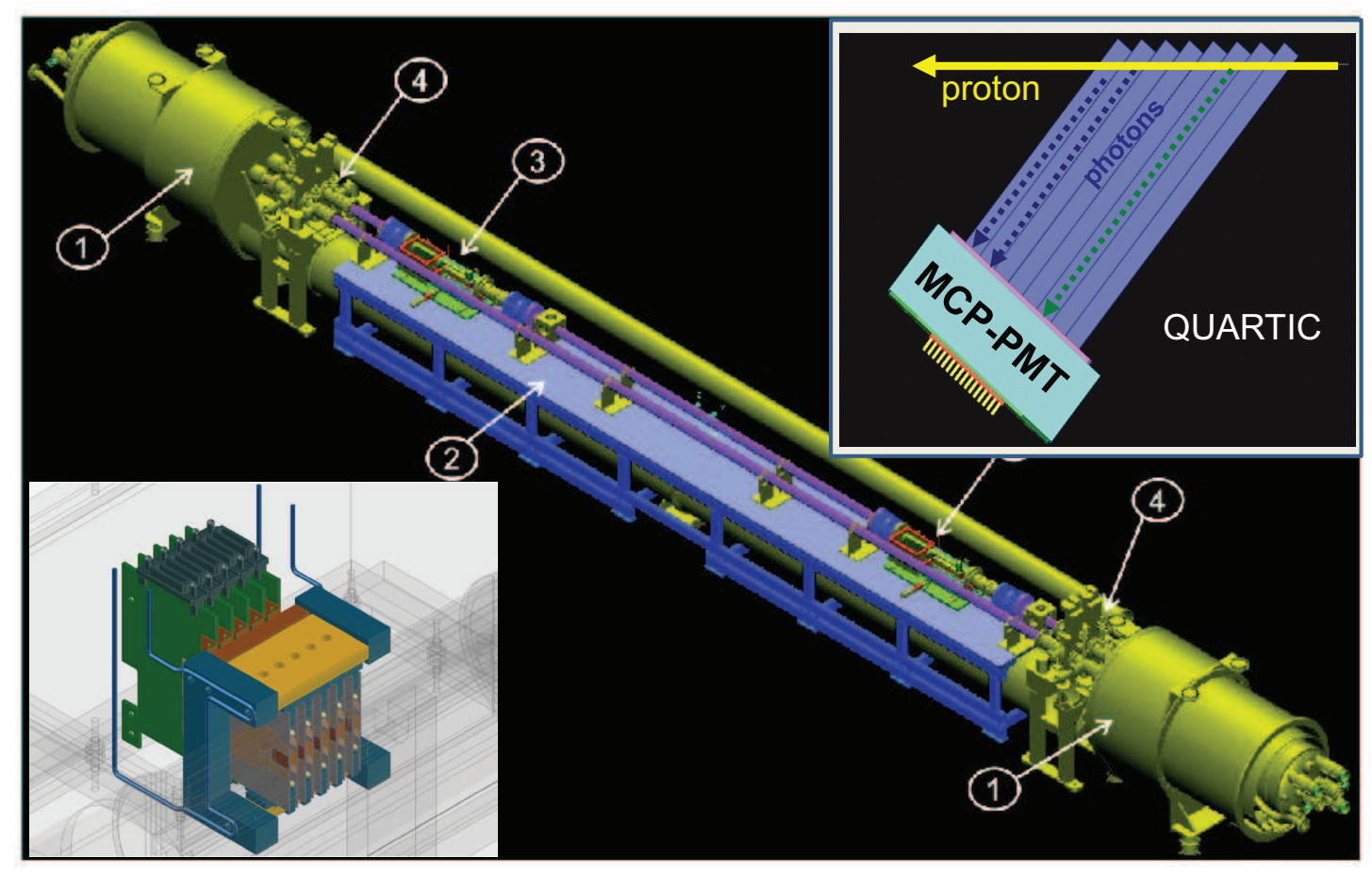

Figure 2: A modified connection cryostat incorporating a movable beam pipe and pockets for AFP timing detectors (upper right inset) and 3-D silicon detectors (lower left inset) for the AFP $420 \mathrm{~m}$ stations.

\section{References}

[1] The ATLAS Collaboration, G. Aad et al., The ATLAS Experiment at the CERN Large Hadron Collider, JINST 3 (2008) S08003.

[2] "Charged-particle multiplicities in pp interactions at $\sqrt{s}=900 \mathrm{GeV}$ measured with the ATLAS detector at the LHC, Phys Lett B 688, Issue 1, (2010) 21-42.

[3] "The FP420 R\&D Project: Higgs and New Physics with Forward Protons at the LHC," FP420 Collaboration, arXiv:0806.0302v2 [hep-ex], published in J. Inst.:

http://www.iop.org/EJ/abstract/1748-0221/4/10/T10001. 